\title{
Dynamic failure of viscoplastic structures under ASB and micro-voiding
}

\author{
Hannah Lois Dorothy ${ }^{1}$, and Patrice Longere ${ }^{1, *}$ \\ ${ }^{1}$ Université de Toulouse, UT3-UPS, ISAE-SUPAERO, Institut Clément Ader (CNRS 5312), Toulouse, France
}

\begin{abstract}
Adiabatic shear bands are known to cause premature structural failure in high strength metals and alloys. The observation of adiabatic shear bands inside partially fractured specimens evidences the presence of micro-voids as the precursor of the ultimate failure. An enriched model containing the effects of adiabatic shear banding and micro-voiding mechanisms was developed and it is here taken through a calibration procedure. The aim of the present work is to evaluate the performances of the enriched model considering an initial-boundary value problem. To that purpose, the model has been implemented as user material in the engineering finite element computation code LS-DYNA. Numerical simulation of the dynamic shearing of hat shaped structures is conducted and the interest of accounting for the pre-failure stage consisting of micro-voiding in the ASB wake is emphasized.
\end{abstract}

\section{Introduction}

One of the important mechanisms triggering the failure of high strength metals and alloys under high strain rate loading is the adiabatic shear banding (ASB). The adiabatic shear bands are narrow shear strain concentrated regions occurring as the result of thermomechanical instability when thermal softening overcomes strain hardening under conditions of quasiadiabaticity and low stress triaxiality. The shear bands lead to premature material failure and may prove catastrophic when occurring in high strength, lightweight materials, including aluminum and titanium alloys, submitted to accidental overloads. The ASB also finds application in high speed machining in which it helps to reduce the cutting force but at the expense of cutting force oscillation and less smooth surface finish. In the context of engineering design, it is thus crucial to be able to numerically reproduce the consequences of this phenomenon.

A 3D phenomenological model built within irreversible thermodynamics framework was originally developed by [1] to describe the material behavior under ASB induced degradation. Unlike numerously available literature describing ASB, this model is developed in a large scale postulate wherein the representative volume element contains the band amounting to a global insight into the material response. The ASB model accordingly accounts for the anisotropic degradation of the elastic and plastic moduli and a deviation of the viscoplastic flow depending on the band's orientation in space.

According to experimental observations, see e.g. [2] and [3], the fractured surface of dynamically deformed specimens showed evidence of the presence of microvoids in the wake of the shear bands. [4] also report from experiments on hat shaped specimens that micro-cracks and micro-voids are initiated at different places along the shear band. It is thus clearly seen that the fracture occurs due to two successive mechanisms: adiabatic shear banding and then micro-voiding. Therefore [5] and [6] enriched the original constitutive model to include the effects of micro-voiding (MV)-induced degradation. The model at stake is henceforth called enriched ASB+MV model.

The performances of any constitutive model must be evaluated through verification and validation $(\mathrm{V} \& \mathrm{~V})$ procedure as explained in [7]. In the V\&V procedure, the model is first numerically implemented at the scale of a representative volume element (RVE) (or equivalently a single finite element) in order to determine the viscoplasticity and viscous degradation related constants for a given material. The model performance is then evaluated and the constants are further calibrated if need be considering an initial-boundary value problem (IBVP). Here a hat shaped structure (HSS) is used for this step of verification as the ASB path in this structure is known a priori. It is then required to further validate the model on increasingly complex structures, for example, ballistic impact, high speed machining, etc. The procedure has already been carried out on the constitutive ASB model describing the ASB effect only ([8] [9]). It is now required to calibrate the enriched ASB+MV model. Due to the challenge of obtaining material constants relative to the micro-voiding, rather than constants identification, a model feasibility study has been conducted at the RVE scale in [6].

The aim here is to evaluate the performances of the enriched ASB $+\mathrm{MV}$ model considering a complex IBVP. To that purpose, the model has been implemented as user material in the engineering finite element computation

* Corresponding author: patrice.longere@isae.fr 
code LS-DYNA. Numerical simulation of the dynamic shearing of hat shaped structures is conducted. The interest of accounting for the pre-failure stage consisting of micro-voiding in the ASB wake is emphasized.

Section 2 outlines the main features of the enriched ASB + MV model whose performances are evaluated in Section 3 via the numerical simulation of the dynamic loading of a hat shaped structure. Conclusions are drawn in Section 4.

\section{Enlarged ASB+MV model}

This section outlines the salient features of the enlarged ASB+MV constitutive model and also states the modifications incorporated to the original model found in [5] and [6].The aim of the enriched model is to clearly distinguish the contributions to the degradation coming from the ASB on the one hand and the MV on the other hand.

As mentioned in the introduction, the model is developed in a large scale postulate wherein the representative volume element contains the band, and not the opposite as usually done. The shear band is strongly oriented in space and it induces an anisotropic degradation of the elastic and plastic moduli of the volume element based on its orientation. The material outside the band exhibits a "regular" viscoplastic behavior and the material inside the band is termed "singular" exhibiting reduced strength. The vectors n and $\mathbf{g}$ are the normal and the tangent to the ASB orientation respectively and they define the structural tensors $\quad \mathbf{N}=\mathbf{n} \otimes \mathbf{n} \quad$ and $\quad \mathbf{M}=\operatorname{sym}[\mathbf{g} \otimes \mathbf{n}] . \quad$ The degradation induced by the ASB and subsequently the $\mathrm{MV}$ in its wake is therefore defined by the second order tensor anisotropic variable $\mathbf{D}$ with $\mathcal{D}$ being the degradation intensity.

$$
\mathbf{D}=\mathcal{D} \mathbf{N}
$$

The yield function $\mathrm{F}$ of the enlarged $\mathrm{ASB}+\mathrm{MV}$ model reads:

$$
\mathrm{F}=\sigma_{e q}+3 \xi \Delta G_{M V}^{2} \exp \left(\frac{\sigma_{m}}{\bar{\sigma}}\right)-\left(R_{0}+r\right)=\sigma_{v} \geq 0
$$

where $\sigma_{e q}$ is the transformed equivalent stress defined later, $\left(R_{0}+r\right)$ the rate independent yield stress with $r$ the strain hardening force, $\sigma_{m}$ the mean stress and $\bar{\sigma}$ a reference stress, $\sigma_{v}$ the strain rate induced overstress. $\mathbf{G}$ is the deterioration driving force conjugate to $\mathbf{D}$ and contained in $\Delta G_{I}(I=\mathrm{ASB}, \mathrm{MV})$. The latter is the difference between the current value of $\mathbf{G}$ and its value at the onset of the corresponding mechanisms in question (ASB then MV) projected on the band direction, namely $G_{I}=\mathbf{G}_{I}: \mathbf{N}$. The second term of the expression of F containing $\sigma_{m}$ corresponds to the expansion of microvoids. The $\Delta G_{M V}$ in this term has been modified to
$\Delta G_{M V}^{2}$ from the previous work [5] and [6] in order to be consistent with the MV incipience while deriving the damage rate expressed in $(5)_{2}$.

A transformed equivalent stress $\sigma_{e q}$ is employed here which comprises the regular plastic von Mises equivalent stress $\sigma_{V M}$ and the singular stresses induced by ASB - $\sigma_{A S B}$ and then by MV- $\sigma_{M V}$. The expressions for the $\sigma_{e q}$ and its components are

$$
{\sigma_{e q}}^{2}={\sigma_{V M}}^{2}+{\sigma_{A S B}}^{2}+{\sigma_{M V}}^{2}
$$

where

$$
\begin{aligned}
& \sigma_{V M}=\sqrt{\frac{3}{2} \mathbf{s}: \mathbf{s}} \\
& \sigma_{A S B}=\sqrt{\eta_{A S B} \Delta G_{A S B}^{2}\left(3 \tau^{2}+\alpha\left\langle\sigma_{N}\right\rangle^{2}\right)} \\
& \sigma_{M V}=\sqrt{\eta_{M V} \Delta G_{M V}^{2}\left(3 \tau^{2}+\beta\left\langle\sigma_{N}\right\rangle^{2}\right)}
\end{aligned}
$$

In this paper, the loss of strength of the material due to $\mathrm{ASB}$ and the subsequent $\mathrm{MV}$ in the direction normal to the band during tension loading is considered by adding the normal stress $\sigma_{N}$ term in the expression of the transformed equivalent stress. $\langle$.$\rangle defines Macauley$ brackets. $\tau$ is the shear stress resolved along the band plane defined later.

In addition to describing the material effect of degradation, the model also accounts for the deviation of the viscoplastic flow due to the presence of ASB and MV. This is demonstrated by the total inelastic strain rate $\mathbf{d}^{\text {in }}$ which is composed of the regular plastic and singular (ASB+MV) plastic strain rates $\mathbf{d}^{i n(A S B)}$ and $\mathbf{d}^{i n(M V)}$. The inelastic strain and deterioration rates are derived from the plastic potential $\mathrm{H}=\mathrm{F}\left(\left\langle\sigma_{N}\right\rangle \equiv 0\right)$, see (2) and (4), according to the normality rule (non associate plasticity) as follows:

$$
\begin{aligned}
& d_{i j}^{i n}=\Lambda_{p} \frac{\partial \mathrm{H}}{\partial \sigma_{i j}}=d_{i j}^{i n}(P P)+d_{i j}^{i n}(A S B)+d_{i j}^{i n}(M V) \\
& D_{i j}=\Lambda_{d} \frac{\partial \mathrm{H}}{\partial G_{i j}}=D_{i j}^{\nabla}+\underset{\nabla S B}{\nabla}+D_{i j}^{M V}
\end{aligned}
$$

where $\Lambda_{p}$ and $\Lambda_{d}$ are viscoplasticity and viscousdeterioration multipliers respectively. The detailed rate equations are shown below where $\eta_{A S B}$ and $\eta_{M V}$ are degradation related constants, $\mathbf{S}$ is the deviatoric part of the stress tensor, $\tau=\mathbf{s}: \mathbf{M}$ is the resolved shear stress and $\mathbf{I}$ the identity tensor. 


$$
\left\{\begin{array}{l}
d_{i j}^{i n(P P)}=\frac{3}{2} \Lambda_{P} \frac{s_{i j}}{\sigma_{e q}} \\
d_{i j}^{i n(A S B)}=3 \Lambda_{P} \frac{\eta_{A S B} \Delta G_{A S B}^{2} \tau}{\sigma_{e q}} M_{i j} \\
d_{i j}^{i n(M V)}=3 \Lambda_{P}\left[\frac{\eta_{M V} \Delta G_{M V}^{2} \tau}{\sigma_{e q}} M_{i j}+\frac{\xi}{3 \bar{\sigma}} \Delta G_{M V}^{2} \exp \left(\frac{\sigma_{m}}{\bar{\sigma}}\right) I_{i j}\right]
\end{array}\right.
$$

and

$$
\left\{\begin{array}{l}
\nabla_{i j}^{A S B}=\frac{3 \tau^{2}}{\sigma_{e q}} \Lambda_{A S B} \eta_{A S B} \Delta G_{A S B} N_{i j} \\
\nabla \\
D_{i j}^{M V}=\left[\frac{3 \tau^{2}}{\sigma_{e q}} \Lambda_{M V} \eta_{M V} \Delta G_{M V}+3 \Lambda_{M V} \xi \exp \left(\frac{\sigma_{m}}{\bar{\sigma}}\right)\right] N_{i j}
\end{array}\right.
$$

As it remains a challenge to identify the MV related material constants from the experiments, a set of arbitrary constants are tentatively used for the numerical simulation. The feasibility of the model on a representative volume element scale has been demonstrated in [6] for shear loading. The main results are presented here for the enriched model with the modifications made. Fig. 1 shows the shear stress-shear strain curve for three different cases: model with plasticity only, model with plasticity and ASB induced degradation, model with plasticity and ASB and subsequent MV induced degradation. The plasticity model shows no drastic material strength reduction except for the thermal softening. The following are the various stages of the stress-strain curve for the other two models. At first, the material is subjected to strain hardening and the plastic dissipation induced rise in regular temperature causes thermal softening. Then a competition occurs between the hardening and softening mechanisms. Soon after thermal softening overcomes the strain hardening the ASB initiates. A linear perturbation method is used to evaluate the ASB incipience (see [1] for details). The ASB evolution causes a drop in the shear strength of the material. The model with ASB+MV exhibits supplementary material degradation due to the additional MV mechanism, as expected.

The trace of the degradation tensor $\mathbf{D}$ is plotted in Fig.2 for the cases with ASB only and ASB+MV. The additional contribution of the $\mathrm{MV}$ to the degradation once it initiates is clearly visible.

The kinematic consequence of the presence of ASB $+\mathrm{MV}$ is demonstrated in Fig. 3 by the different components of the total inelastic strain rate in $(5)_{1}$. It is initially composed of the regular plastic strain which then diminishes paving the way for the singular plastic strain rate to take predominance. This singular plastic strain rate is first composed of ASB induced strain rate and then MV induced strain rate.

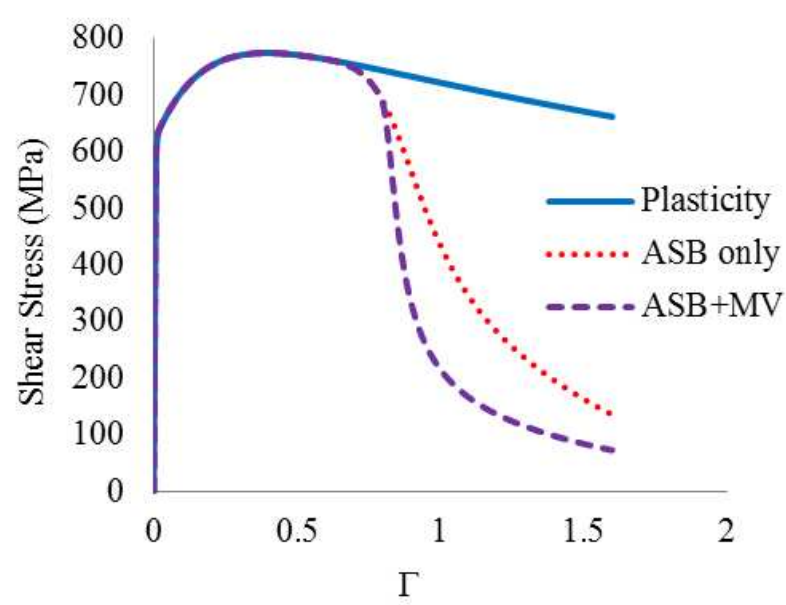

Fig.1. Shear stress-shear strain curve for three different cases: plasticity only, ASB induced degradation, ASB and subsequent MV induced degradation. Volume element under shear loading.

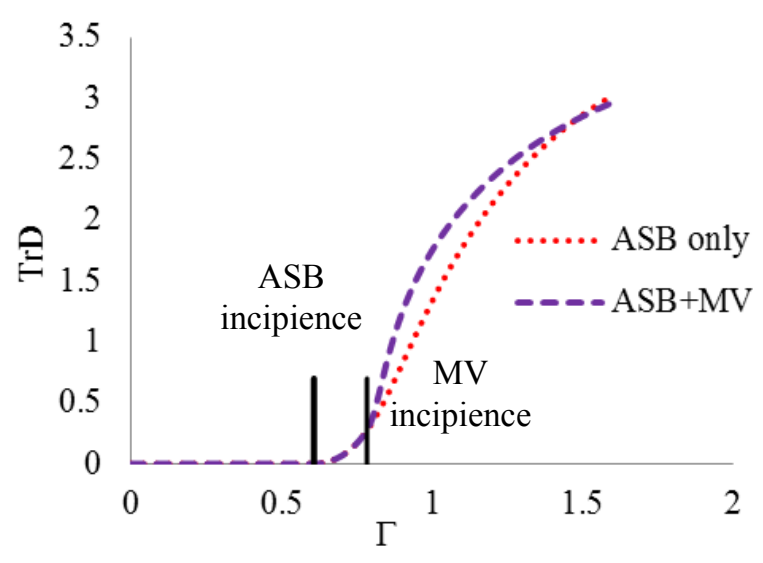

Fig.2. Degradation variable-shear strain curve for two different cases: ASB induced degradation, ASB and subsequent MV induced degradation. Volume element under shear loading.

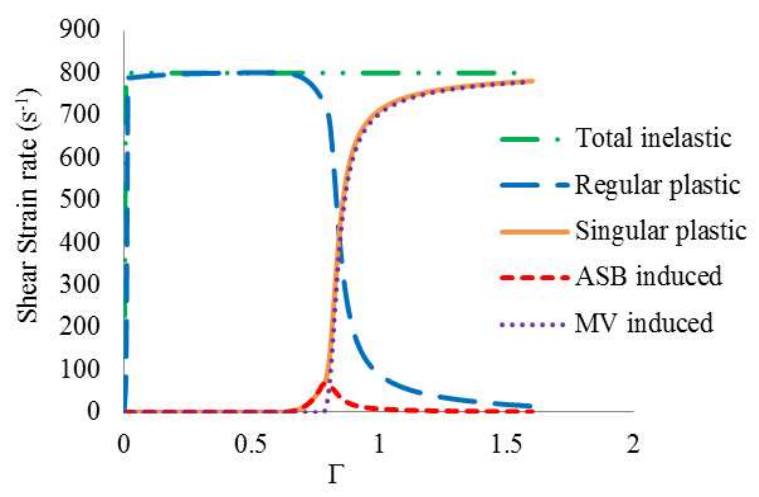

Fig.3. Shear strain rate-shear strain curve for two different cases: ASB induced degradation, ASB and subsequent MV induced degradation. Volume element under shear loading.

The model is thus demonstrated to be feasible on a representative volume element scale. 


\section{Numerical structural scale}

\section{implementation}

on

Following the $\mathrm{V} \& \mathrm{~V}$ procedure, it is now required to implement the enriched ASB+MV model on a structural scale. A hat shaped structure is used to this end. The HSS is impacted in a direct Hopkinson bar set up as shown in Fig.4 and the gage section of the HSS experiences combined compression and shear facilitating the formation of ASB. The same set of material constants used in the RVE scale simulation in Section 2 is employed here.

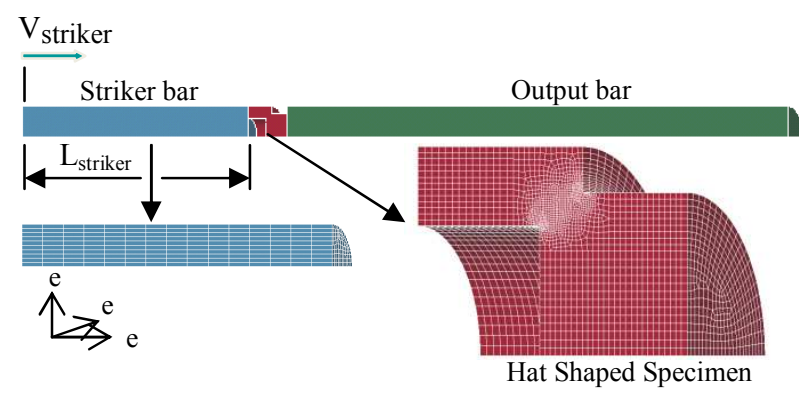

Fig.4. Direct impact experimental set-up considered for the numerical simulation.

Certain elements from the gage section shown in Fig. 5 are chosen to plot the following curves.
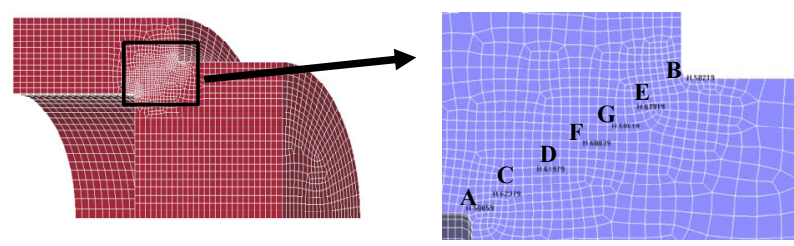

Fig.5. Detailed view of the meshed hat shaped structure with the location of the finite elements in which quantities are extracted.

In Figs. 6-7, the resolved shear stress $\tau$ is plotted as a function of time for the models with ASB only and ASB+MV respectively. As in the case of the RVE simulation, a mild drop in strength is observed for the case of ASB only and a more significant drop in strength for the ASB+MV case.

The evolution of the degradation variable $\mathbf{D}$ for ASB and ASB+MV is shown in Figs.8-9 respectively. The case of ASB+MV shows significant addition to the degradation intensity when compared to ASB only case, as expected.

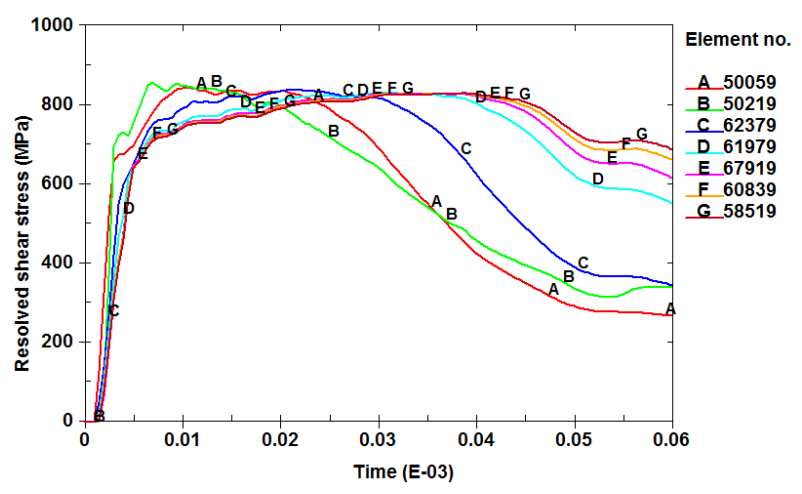

Fig.6. Resolved shear stress history in finite elements located inside the strip of intense shearing, see Fig.5. Configuration with ASB only. Numerical simulation of the dynamic loading of hat shaped structure.

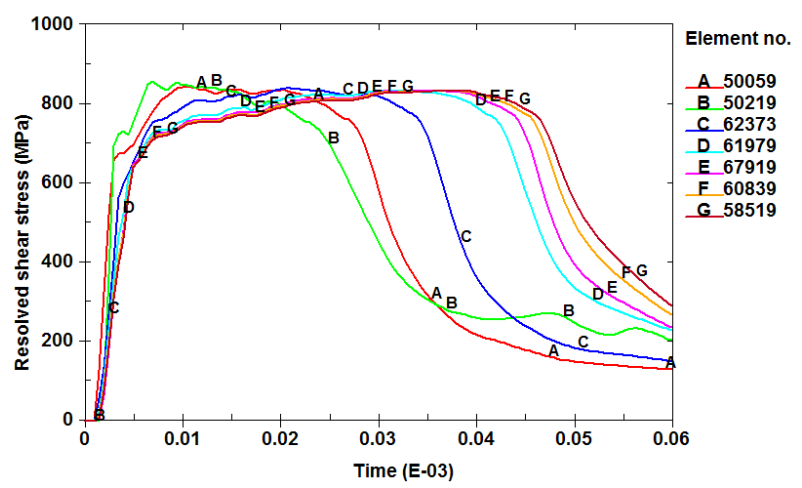

Fig.7. Resolved shear stress history in finite elements located inside the strip of intense shearing, see Fig.5. Configuration with ASB+MV. Numerical simulation of the dynamic loading of hat shaped structure.

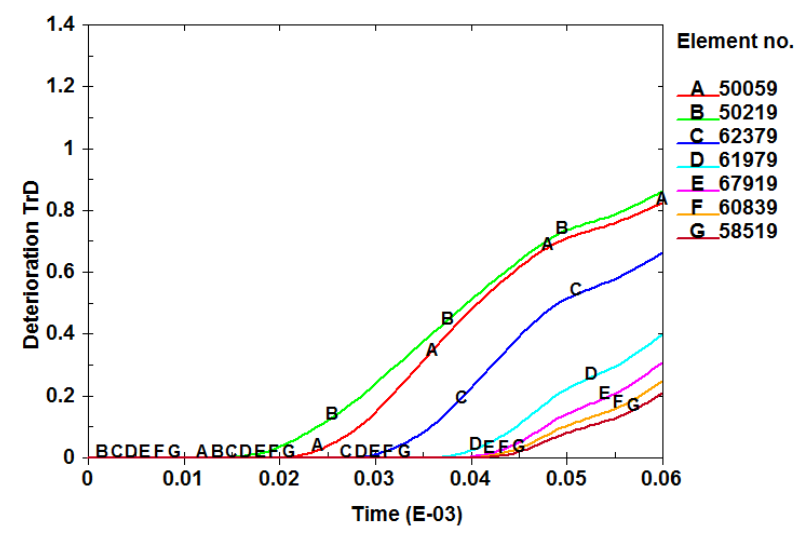

Fig.8. Degradation variable history in finite elements located inside the strip of intense shearing, see Fig.5. Configuration with ASB only. Numerical simulation of the dynamic loading of hat shaped structure. 


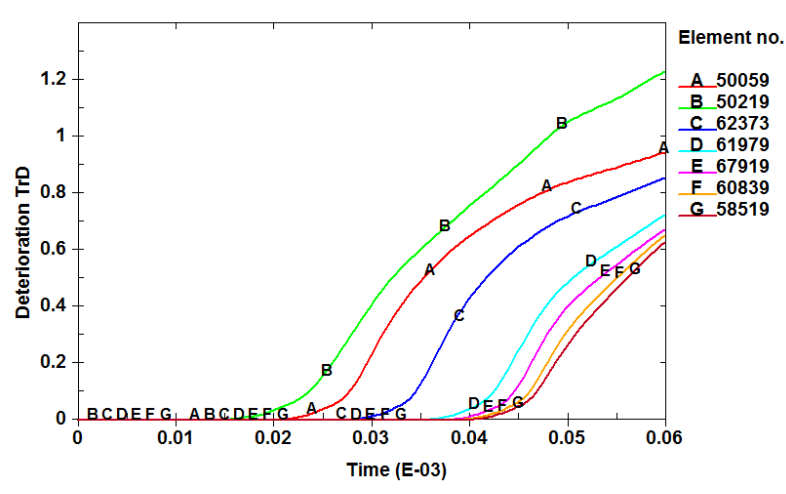

Fig.9. Degradation variable history in finite elements located inside the strip of intense shearing, see Fig.5. Configuration with ASB + MV. Numerical simulation of the dynamic loading of hat shaped structure.

The numerical deterioration map gives an indication of the presence of $\mathrm{ASB} / \mathrm{MV}$ in the finite elements. In Fig. 10 for the case of ASB only, the elements in red/dark are subject to ASB whereas the elements in green/light are not. Fig.11, corresponding to the configuration with ASB+MV, shows the elements subject to ASB (left, elements in red/dark) and the elements subject to MV (right, element in magenta/dark).

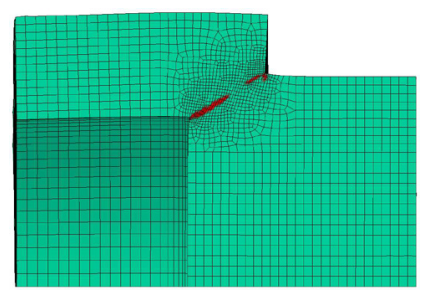

Fig.10. Map of the degradation variable indicator. Elements in red/dark are subject to ASB. Configuration with ASB only. Numerical simulation of the dynamic loading of hat shaped structure.
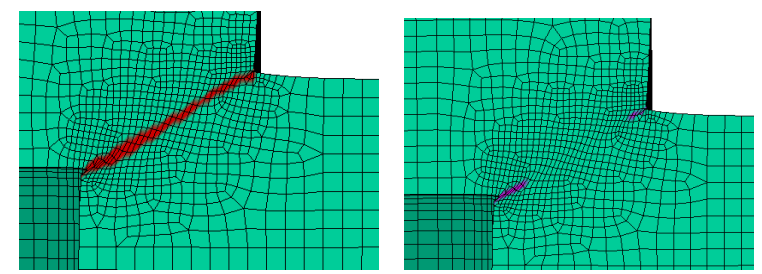

Fig.11. Maps of the degradation variable indicators. Left: ASB-induced degradation indicator, elements in red/dark are subject to ASB. Right: MV-induced degradation indicator, elements in magenta/dark are subject to MV. Configuration with ASB+MV. Numerical simulation of the dynamic loading of hat shaped structure.

The model is thus demonstrated to be numerically robust on the structural scale. In order to complete the $\mathrm{V} \& \mathrm{~V}$ procedure, the next step is to implement the model on a more complex IBVP such as the high speed machining which is a work in progress.

Further enrichments of the model could be carried out in terms of considering other softening mechanisms such as dynamic recrystallization, see [10], which has been shown to have significant influence on the ASB incipience when combined with thermal softening for e.g. high strength titanium alloy, see [11].

\section{Conclusion}

The enriched model describing the adiabatic shear banding and micro-voiding mechanisms has been subjected to slight modifications in order to be more consistent with the physics of the shear localization and subsequent ductile damage process. The model performances are evaluated within a calibration procedure. Following this procedure, the model has been demonstrated to be numerically feasible at the representative volume element scale and also on a structure namely the hat shaped structure. The challenge remains to experimentally identify the micro-voiding related constants. In order to complete the validation process, the model has to be evaluated on a complex initial boundary value problem such as high speed machining, which is a work in progress. The constitutive model could be further enriched by implementing other softening mechanisms such as dynamic recrystallization.

\section{References}

1. Longère P., Dragon A., Trumel H., de Resseguier T., Deprince X. and Petitpas E., Modelling adiabatic shear banding via damage mechanics approach, Arch. Mech., 55, pp.3-38 (2003)

2. Odeshi A.G., Al-ameeri S., Mirfakhraei S., Yazdani F., Bassim M.N., Deformation and failure mechanism in AISI 4340 steel under ballistic impact, Theor. Appl. Fract. Mech., 45, pp.18-24. (2006)

3. Longère P., Dragon A., Dynamic vs. quasi-static shear failure of high strength metallic alloys: Experimental issues, Mech. Mat. 80 203-218 (2015)

4. Peirs J., Verleysen P., Degrieck J., Coghe F., The use of hat-shaped specimens to study the high strain rate shear behaviour of $\mathrm{Ti}-6 \mathrm{Al}-4 \mathrm{~V}$, Int. J. Impact Eng., 37, pp. 703-714 (2010)

5. Longère P., Dragon A., Enlarged finite strain modelling incorporating adiabatic shear banding and post-localization microvoiding as shear failure mechanisms, Int. J. Dam. Mech., 25, 1142-1169 (2016)

6. Dorothy H.L., Longère P., Dragon A., Coupled ASB-and-microvoiding-assisted dynamic ductile failure, Proc. Eng.. 197, pp.60-68 (2017)

7. Longère P., Adiabatic shear banding assisted dynamic failure: Some modeling issues, Mech. Mat., 116, pp.49-66 (2018)

8. Longère P., Dragon A., Trumel H. and Deprince $\mathrm{X}$., Adiabatic shear banding induced degradation in a thermo-elastic/viscoplastic material under dynamic loading, Int. J. Impact Eng, 32, pp.285320 (2005)

9. Longère P., Dragon A., Deprince X., Numerical study of impact penetration shearing employing 
finite strain viscoplasticity model incorporating adiabatic shear banding, J. Eng. Mat. Tech., ASME, 131, pp.011105.1-14 (2009)

10. Rittel D., Landau P., Venkert A., Dynamic recrystallization as a potential cause for adiabatic shear failure, Phys. Rev. Lett. 101, 165501(2008)

11. Longère $P$., Respective/combined roles of thermal softening and dynamic recrystallization in adiabatic shear banding initiation, Mech. Mat., 117, pp. 8190 (2018) 